\title{
Are randomized clinical trials necessary in cancer surgery?
}

\author{
Krzysztof Bielecki
}

Change alone is eternal, perpetual, immortal

Arthur Schopenhauer

Good judgment comes from experience

and experience comes from bad judgment

The article outlines the main principles of randomized clinical trials (RCTs) and discusses various problems related to their organization and performance. RCTs constitute a golden standard in medical literature, including surgical journals, but when badly constructed and conducted, they might cause more harm than good. The chief difficulties involved in organizing RCTs include the appropriate selection of patients, correct randomization, serious ethical concerns, and considerable cost. Their presence is attested to by the negligible number of RCTs, which only represent as few as 3 to $7 \%$ of all surgical studies. Cancer surgery is often radical and irreversible and requires respect for the preferences of patients and surgeons. It is not a lab experiment where everything can be neatly planned and carried out. In RCTs, study groups differ in terms of one variable. Cancer surgery, on the other hand, needs to take into account many different variables describing the patient, the disease, and the surgeon at the same time. The results of randomized studies cannot be applied across the board, even for patients who suffer from the same cancer. The findings are true only for the group of patients who participate in the study and their generalization to the whole patient population raises a serious objection.

There is a great need for clinical studies in cancer surgery, no matter whether they are randomized or not. Large, prospective, multi-center cohort studies, well-kept cancer registries, retrospective studies, and clinical case series furnish a very useful and precious source of knowledge. Expert consensus conferences are particularly valuable in practical terms. In recent years, there has been increasing pressure on reference centers from the medical industry and business to perform randomized trials. Studies of this kind, especially when published in high-impact journals, play an important role in introducing new equipment, methods, and drugs to clinical practice.

This causes a reasonable objection. Something must be "afoot" if all studies are currently required to feature a conflictof-interests (COI) formula. To sum up, cancer surgery needs scientific studies, but not necessarily only RCTs. The author is against the overemphasis on randomized trials and the impact factor in clinical practice.

NOWOTWORY J Oncol 2016; 66, 6: 494-498

Key words: randomized trials, difficulties in carrying out, controlled studies, national cancer registries, ethical issues, surgical studies, evidence- and preference-based medicine 


\section{Introduction}

Until recently, doctors would treat their patients "according to their best knowledge and conscience". Article 4 of the Medical Code of Ethics stipulates that "in execution of his/her duties the physician must be free to proceed in accordance with his/her conscience and to undertake professional activities in accordance with contemporary medical knowledge".

Today, we rely on available economic and technological solutions, treatment benefits, clinical wisdom, common sense, prudence, and above all, human empathy and the good of the patient. In this context, it is difficult to conduct randomized clinical trials and one may even ask whether they are necessary at all [1].

Cancer surgery usually causes an irreversible clinical change. Thus, creating placebo control groups raises serious ethical reservations. In a randomized trial, we may operate on a group of patients and get a negative; the trial will be stopped, of course, but who will be accountable to patients who already suffer from serious complications? Are we going to say that whatever happened, happened for the sake of science, at the service of evidence-based medicine?

"Prospective, randomized, double-blind, placebo-controlled trials" are now the magic formula for some physicians. The evidence and knowledge obtained through such studies have been expected to furnish the basis for clinical proceedings for the past 20 years or so, or at least this is what scientists claim. In 1996, R. Horton, editor-in-chief of the Lancet, wrote "Only when the quality of publications in the surgical literature has improved will surgeons reasonably be able to rebut the charge that as much as half of the research they undertake is misconceived" [2]. D.L. Sackett, the pioneer of evidence-based medicine, stated that: "As physicians, whether serving individual patients or populations, we have always sought to base our decisions and actions on the best possible evidence" [3]. The best possible evidence comes from prospective, randomized, placebo-controlled studies; these should also inform decisions in surgery, which often has irreversible consequences.

In cancer surgery, RCTs should not be conducted if participants cannot be ensured the top standard of treatment in terms of organization, methodology, funding, follow-up, and patient survival rates, as well as adequate handling of possible complications. Neither are they a good choice if important implications from stage 1 and 2 trials, ethical, medical, statistical or other factors fail to justify the need for a randomized study.

Before conducting an RCT, it is worth asking: cui bono? Is the good of the patient really a priority for the medical institution that organizes and funds the study? Will we be able to track patients in the follow-up to the trial? The chief elements to consider include:

- reduced mortality rate;
- increased average survival rate;

— increased five-year survival rate;

— improved quality of life;

- improved cost/effect ratio, e.g. the cost of one-year survival.

Even though thoroughly planned and well-conducted RCTs provide the most precise and reliable information, they are still quite rare in cancer surgery. A survey of literature published in the 1990s indicated that they accounted for as few as $7 \%$ of all trials performed in the field [4]. In 2003, experts assessed that only $3.4 \%$ of studies published in leading surgical journals involved RCTs [5]. In addition, only $44.1 \%$ of RCTs compared and contrasted different surgical methods, while $55.9 \%$ focused exclusively on conservative treatment [5].

In 1985, 14\% of all studies published in the British Journal of Surgery were randomized; seven years later, the figure dropped to just 5\% [6]. There are many methodological and organizational reasons for this decrease. The main obstacle to such trials is the clear preference of surgeons and patients for specific methods [4]. Lack of funding and patient preferences were identified as the most important barriers to RCTs by a group of Australian surgeons [7]. Another study estimated that only around $45 \%$ of patients who met the qualification criteria agreed to take part in the study, while $42 \%$ of colorectal cancer patients preferred one of the studied surgical methods [8].

Difficulties are posed not only by the preferences of patients, but also those of surgeons. A concept of community equipoise was introduced to evaluate discrepancies in expert opinions, preferences, and assessment of new methods; the idea refers to the homogenization of beliefs, i.e. a community equilibrium among experts that makes randomized studies possible. In many surgical cases, there is no data from randomized clinical trials and the best treatment is chosen on the basis of clinical guidelines that take patient preferences into account. For instance, doctors opt for a less radical and invasive method that ensures a potentially lower survival rate, but a better quality of life. The decision is consulted with the patient.

It is possible to evaluate the preferences of the surgeon community, e.g. during conferences attended by experts who decide on specific recommendations. Such consensus relies not only on the knowledge obtained through RCTs but also the preferences of surgeons that are rooted in their experience.

\section{Why should we conduct randomized trials?}

RCTs originated as a prevalent phenomenon in the last decades of the $20^{\text {th }}$ century. Previously, clinicians based their decisions on observations carried out over many years, as well as the observed efficacy of specific methods. Many treatments, such as insulin, vaccinations, or appendectomy 
in acute appendicitis, do not require randomized trials. Nobody expects clinical studies to be conducted where effective treatments are already available.

Certain modifications in cancer surgery slightly improve treatment results and raise the quality of life, but is that reason enough to organize difficult and costly randomized trials that take years and years to complete? Informed by the fashion for EBM, we now accept that RCTs are the most valuable source of knowledge, but we also need to recognize their flaws and difficulties. They are time-consuming in terms of planning, patient selection, performance, and monitoring. Their findings cannot be generalized and universally applied since they are true for a small group selected in accordance with specific eligibility criteria. Whenever a rare disease is investigated or desired results show up only after a long time, RCTs are not possible.

Randomized trials are also controversial for ethical reasons. How can surgeons possibly accept randomization knowing that one of the studied treatment methods might be better than the other, even if that belief is founded on untested, anecdotal evidence?

Methodological problems are of great concern as well, especially in surgery. How should we go about evaluating the learning curve for a new treatment, whose efficacy we want to test against an existing method? Surgeons need to achieve mastery in a new technique before they can begin to recruit patients. And what is the situation like today? A new method appears, and before we even know how to use it, a company that produces relevant surgical equipment suggests a randomized clinical trial should be conducted.

In order to reduce systemic errors, careful attention should be paid to choosing the randomization method, allocation concealment, and properly blinding the study. In surgical studies, blinding is very difficult, especially for the surgeon. For instance, when we compare laparoscopic and traditional procedures, the cat is let out of the bag at the first dressing of the wound. In order to properly carry out a RCT, a sham operation needs to be performed in the control group, which raises serious ethical concerns and may cause health complications, etc. The blinding process should no doubt cover patients, the personnel who collects patient data, administrative staff, and all those responsible for funding and results analysis. If this is not possible, RCTs should not be conducted. The good of the patient should be taken into account in terms of treatment intentions; it is crucial to draw up and carry out a detailed and complete follow-up program and strive to minimize statistical error by defining the precise size of studied patient groups (power analysis) [9].

\section{The greatest problems with RCTs are related to patients}

Surgical procedures are irreversible. We know, for instance, that in rectal cancer, lower anterior resection is con- sidered superior to abdominal-sacral incision, because it does not end in a definitive stoma. However, some authors point out that anterior resection involves a higher rate of relapse. How can we persuade patients to take part in the study, knowing that they may be assigned to a group that undergoes a definitive stoma, if it could be avoided if they ended up in the group treated by means of anterior resection [10]?

Another reason for such a small number of RCTs in cancer surgery is the lack of legal regulations concerning randomized trials in many countries. There are few institutions such as the US Food and Drug Administration or the Canadian Health Protection Branch that would oversee clinical drug trials. Clinicians introduce new surgical methods with a certain apprehension and many restrictions even before their local ethics committees. A good example of this lack of control was the "explosion" of laparoscopic techniques that occurred both in general and cancer surgery without any preliminary controlled studies. Most surgeons simply jumped on the bandwagon, because they were new, faster, and more cost-effective.

All these factors do not make an exhaustive list of the issues and difficulties that make surgeons look at RCTs with a certain reserve. Most prefer to rely on the results of large long-term observational studies. Randomized clinical trial results available in cancer surgery are few and far between and their statistical quality is low, estimated at 0.4 on a scale from 0 to 1 [11]. What we should do instead, therefore, is look for prospective, pragmatically well-controlled comparative and observational studies [9]. Their informational value is far greater than that of badly planned and conducted RCTs.

In 1996, T. Quill published an alarming article discussing how the responsibility for medical procedures has been increasingly moved from the physician to the patient in the ostensible interest of the autonomy of the latter [12]. There is a serious clash between the huge store of knowledge possessed by the physician and the expectations of patients, whose grasp of medicine is at best negligible. The old question thus comes up again: what should matter more in clinical decision-making - the will or the good of the patient?

\section{Surgeons do not need to live with the consequences of treatment - patients and families do}

Table I presents two alternative scenarios for clinical medical proceedings: A. evidence-based medicine, B. preference-based medicine.

Both scenarios help establish a relationship between the doctor and the patient that works best for the latter. The doctor is an expert on medicine, patients and their relatives are experts on patient preferences and expectations. Preference-based medicine thus relies on the evidence they provide regarding the reality and objectives of treatment, its 
Table I. A balanced approach to achieving the goal — targeted patient care

\begin{tabular}{|c|c|}
\hline Evidence-based medicine & Preference-based medicine \\
\hline 1. Defining and asking the right question & 1. Designing and holding an interview with the patient and the family \\
\hline 2. Finding evidence necessary to solve the problem & $\begin{array}{l}\text { 2. Getting the patient and the family to articulate their preferences, } \\
\text { discussing the goal of treatment, presenting the doctor's experience } \\
\text { in non-scientific language }\end{array}$ \\
\hline 3. Critically evaluating the evidence & 3. De-biasing and preventing emotional reactions \\
\hline 4. Taking a decision & $\begin{array}{l}\text { 4. Creating recommendations and looking for consensus (combined } \\
\text { with EBM evidence). }\end{array}$ \\
\hline 5. Assessing feasibility and effectiveness & 5. Ensuring continuing care and follow-up \\
\hline
\end{tabular}

benefits, burdens, and patient's abilities (there is no room for those variables in RCTs). Patients, families, and doctors must arrive at a consensus concerning the method of treatment. The likelihood of a cure should not be exaggerated; nor should the patient be scared with possible complications. It is necessary to put aside enough time for conversation [13].

Clinical trials can be divided into several groups depending on their statistical power (Tab. II). Ellis et al. distinguished three types of evidence:

- evidence from randomized clinical trials;

- evidence from non-randomized, prospective studies and large retrospective studies;

- surgical interventions based on descriptions of clinical cases or results of small clinical trials.

Only $25 \%$ of surgical patients received treatment based on evidence from group 1 [14]. A group of cardiothoracic surgeons who evaluated 50 different procedures reported that only $14 \%$ were based on trials from group 1, and $64 \%$ — group 2 [5]. More than $80 \%$ of all published surgical studies still rely on clinical case studies or small case series from isolated centers.

RCTs are a golden standard today but we must also be aware of the difficulties that they pose in terms of construction and performance in order to make sure that they are

Table II. Levels of evidence used in clinical medicine (website: NHS Research and Development - Center for Evidence-based Medicine)

\begin{tabular}{ll}
\hline Level & Evidence from: \\
\hline $1 \mathrm{a}$ & systematic review of RCTs \\
$1 \mathrm{~b}$ & at least one randomized trial \\
$1 \mathrm{c}$ & all-or-none case series \\
$2 \mathrm{a}$ & systematic review of cohort studies \\
$2 \mathrm{~b}$ & at least one cohort study \\
$2 \mathrm{c}$ & at least one ecological study or "outcomes research" \\
$3 \mathrm{a}$ & systematic review of "case-control" studies \\
$3 \mathrm{~b}$ & individual "case-control" study \\
4 & case series \\
5 & expert opinion \\
\hline
\end{tabular}

really worthwhile and reliable. Otherwise they are bound to do more harm than good. Even if RCTs are conducted, however, the practical implementation of their results will engender another set of problems. The best example are the reports and metaanalyses published by the Cochrane Collaboration, an institution that has now become a platform for publishing top-quality scientific evidence [5].

RCTs have a theoretical advantage over other types of clinical studies, but experimental studies that compare treatment results observed in randomized and non-randomized trials often fail to confirm their superiority, so their obvious (axiomatic) importance cannot be accepted blindly. Small, ill-constructed randomized trials give false results and may do much harm [6]. $56 \%$ of RCTs are reported to suffer from serious methodological errors, and only $58 \%$ are correctly randomized [15].

The history of medicine does not lend support to the statement that only randomized controlled clinical trials can lead to progress in surgery. Once anesthesia and antiseptic drugs were developed, many excellent surgical treatment methods appeared, including those targeted at cancer patients. Many surgeries performed today were introduced long before the advent of randomized trials.

Cancer surgery should rely on so called feasibility studies, i.e. the assessment of the feasibility of a new surgical method or a modification of an existing technique. Such studies allow to compare, for instance, between manual and stapled colorectal anastomosis.

The vagaries of evidence-based medicine are discussed by Winek et al., authors of a very interesting study that encourages deep reflection on the subject [16]. Its final sentence has a particularly strong resonance for me: "Even though contemporary medicine is based on facts, it still remains an art".

\section{Conclusions}

In 2015, the European Colorectal Surgery Congress in St. Gallen featured a panel session devoted to scientific studies in cancer surgery. There has been some fatigue with evidence-based medicine and the exaggerated weight given to RCTs. More and more emphasis is currently placed 
on the importance of well-kept national disease registries and large observational studies.

Success in cancer surgery does not depend on this or that randomized study. In my opinion, it has more to do with patient factors (age, co-morbidities, nutrition, etc.), cancer-related determinants, especially its progression, surgeon characteristics (skill, experience, personality), available technology, and the possibility of combined treatment.

One more thing: medicine is gradually being taken over by the law of material value. Practical assessments of treatment profitability are being tolerated today and a price tag is increasingly put on human life. Doctors are instructed to take decisions based on economic factors. Let us remember that medical economism is immoral, and morality is a categorical and ultimate value that also science should respect. Moral concerns may be hidden from scientific and biological studies, which is why the language of science avoids concepts such as morality and conscience, but we still have a valid code of ethics. Ethics and morality are especially important when planning and conducting scientific studies. There has been a dynamic growth in technology and basic sciences in medicine. Business and industry have caught on to the opportunity of making large profits under the banner of care and the good of the patient. Business has repeatedly attempted to influence scientific studies, which is why many trials, especially RCTs, feature an abbreviated formula $\mathrm{COI}$ - conflict of interest, as well as disclosure statements, sources of funding, etc. Something is afoot after all!

\section{Are randomized clinical trials needed in cancer surgery?}

Yes, but only on the condition that they are correctly planned and conducted. Randomized trials should not crowd out cohort studies, observational, prospective, and retrospective studies. "Science limits itself to stating the facts, but does not determine what is and what it is supposed to be like" [17]. When not conducted properly, even the best-designed study will do more harm than good.

I cannot categorically deny the need for randomized trials in cancer surgery. They are as needed as any other wellplanned and conducted clinical studies. Let us consider the way in which patients are prepared for surgery. It has been a standard procedure to bathe the patient the day before or immediately prior to surgery with the use of antiseptic soap, usually containing chlorhexidine. Metaanalyses of randomized trials have shown that the practice does not reduce the risk of infection [18]. However, will anyone decide to skip bathing for that reason? A clinical study on one side, and clinical experience on the other will determine the choice of action.

"When no evidence achieves the expected level of statistical significance, anecdotal events can often give rise to an interesting hypothesis" [1].

\section{Acknowledgements}

I would like to thank my Friends for their help in preparing this paper, for all their conversations, questions, and statements. My special thanks go to Professor Andrzej Kułakowski, Professor Kazimierz Roszkowski, Professor Andrzej Szawłowski, and Professor Zbigniew Wronkowski.

Conflict of interests: none declared

\section{Professor Krzysztof Bielecki}

Surgical Ward with a Proctology Division

Solec Hospital

Solec 93

00-382 Warszawa, Poland

e-mail:prof.bielecki@gmail.com

Received \& Accepted: 15 Jun 2016

Based on the presentation at the IV Annual Conference of the Nowotwory Journal of Oncology, 'Oncological Debates', held in Warszawa, 8-9th April 2016

\section{References}

1. Maciejewski B. Od Roentgena (120 lat) do stereotaktycznej radiochirurgii. Nowotwory J Oncol 2015; 65: 373-382.

2. Horton R. Surgical research or comic opera: questions but few answers. Lancet 1996; 347: 984-985.

3. Sackett $\mathrm{DL}$, Rosenberg WM. The need for evidence-based medicine $J R$ Soc Med 1995; 88: 620-624

4. Young JM, Salomon MJ, Harrison JD et al. Measuring patient preference and surgeon choice. Surgery 2008; 143: 582-588.

5. Wente MN, Seiler ChM, Uhl W et al. Perspectives of evidence-based surgery. Dig Surg 2003; 20: 263-269.

6. McCulloch $\mathrm{P}$, Taylor I, Sasako $\mathrm{M}$ et al. Randomised trials in surgery: problems and possible solution. BMJ 2002; 324: 1448-1451.

7. Gatterallari M, Ward JE, Solomon MJ et al. Randomized controlled trials in surgery: perceived barriers and attitudes of Australian colorectal surgeons. Dis Colon Rectum 2001; 44: 1413-1420.

8. Solomon MJ, Pager CK, Young JM et al. Patient entry into randomized, controlled trials of colorectal cancer treatment: factor influencing participation. Surgery 2003; 133: 608-613.

9. Farrokhyar F, Karanicolas PJ, Thoma A et al. Randomized controlled trials of surgical interventions. Ann Surg 2010; 251: 409-416.

10. McLeod RS, Wrights JG, Salomon MJ et al. Randomized controlled trials in surgery: issues and problems. Surgery 1996; 119: 483-486.

11. Solomon MJ, MacLeod RS. Should we be performing more randomized controlled trials evaluating surgical operations? Surgery 1995; 118 : 459-467.

12. Quill TE, Brody H. Physician recommendations and patient autonomy: finding a balance between physician power and patient choice. Ann Intern Med 1996; 125: 763-769.

13. QuillTE, Holloway RG. Evidence, preferences, recommendations-finding the right balance in patient care. NEJM 2012; 366: 1653-1655.

14. Ellis J, Mulligan I, Rowe J et al. Inpatient general medicine is evidence based. A-Team, Nuffield Departament of Clinical Medicine. Lancet 1995; 346: 407-410.

15. Black N. Why we need observational studies to evaluate the effectiveness of health care. BMJ 1996; 312: 1215-1218.

16. Winek J, Barańska I, Jodkiewicz Z et al. Dowody, preferencje, rekomendacje. Manowce medycyny faktów - prezentacja przypadku chorego na raka gruczołowego płuc. Nowotwory J Oncol 2015; 65: 428-434.

17. Jonecko A. Fllozofia medycyny w 2000 roku. Acta Med Prem 2001; 21: 29-40.

18. Webster J, Osborne S. Preoperative bathing or showering with skin antiseptics to prevent surgical site infection. Cochrane Database Syst Rev 2012; 9: CD004985. 